\title{
Optical conductivity and $x$-ray absorption and emission study of the band structure of MnN films
}

\author{
S. Granville, B. J. Ruck, F. Budde, A. Koo, J. E. Downes, and H. J. Trodahl \\ MacDiarmid Institute for Advanced Materials and Nanotechnology, School of Chemical and Physical Sciences, \\ Victoria University of Wellington, P.O. Box 600, Wellington, New Zealand \\ A. Bittar, N. Strickland, and G. V. M. Williams \\ Industrial Research Limited, P.O. Box 31-310, Lower Hutt, New Zealand \\ W. R. L. Lambrecht \\ Department of Physics, Case Western Reserve University, Cleveland, Ohio 44106-7079, USA \\ Timothy Learmonth and Kevin E. Smith \\ Department of Physics, Boston University, 590 Commonwealth Avenue, Boston, Massachusetts 02215, USA \\ V. J. Kennedy and A. Markwitz \\ Institute of Geological and Nuclear Sciences, P.O. Box 30368, Lower Hutt, New Zealand \\ Thorsten Schmitt* \\ Laboratory of Materials and Semiconductor Physics, Royal Institute of Technology, Electrum 229, S-164 40 Kista, Sweden
}

(Received 21 July 2005; published 28 November 2005)

\begin{abstract}
The band structure of $\mathrm{MnN}$ films prepared by ion assisted deposition has been investigated by optical conductivity and x-ray absorption and emission spectroscopies. X-ray diffraction and extended x-ray absorption fine structure show the films to be nanocrystalline but phase pure and exhibiting the known antiferromagnetic distorted rocksalt phase. X-ray emission spectroscopy of the $\mathrm{N} K$-edge and x-ray absorption near edge spectroscopy of both the $\mathrm{N} K$ - and $\mathrm{Mn} L$-edges are used to probe the occupied and empty densities of states, which compare well with the $\mathrm{N}(2 p)$ and $\mathrm{Mn}(3 d)$ partial densities of states calculated using the linearized muffin-tin orbital band structure method. A similar comparison is made between the measured optical conductivity and the calculated contribution from interband transitions. It is possible to associate the main features in the measured spectrum with corresponding ones in the calculated optical function. The major differences between calculated and measured spectra can be understood on the basis of a limited electron mean-free-path in these nanocrystalline films, which broadens the features in the joint density of states and relaxes the momentum conservation requirement. The calculated optical functions are analyzed in detail in terms of their dominant band-to-band contributions and in addition the polarization dependence is predicted. Temperature dependent conductivity measurements are also reported and show a clear metallic behavior and a weak Kondolike low temperature anomaly.
\end{abstract}

DOI: 10.1103/PhysRevB.72.205127

PACS number(s): 72.80.Ga, 78.20.Ci

\section{INTRODUCTION}

The transition metal nitrides have gained attention for the extensive range of conduction and magnetic properties they display, as well as for their potential for application in electronic and spintronic technologies. Manganese nitrides are especially interesting, for they form a variety of stoichiometric phases ${ }^{1}$ with $\mathrm{Mn}$ ions in various valence states, and among them one finds both antiferromagnets and ferrimagnets. ${ }^{2-4}$ The Mn nitrides have also generated interest as potentially magnetic secondary phases forming within films of the magnetic semiconductor GaN:Mn, which has been studied as a source of spin-polarized electrons. ${ }^{5,6}$

The most $\mathrm{N}$ rich manganese nitride is the mononitride, $\mathrm{MnN}$, which has an ambient-temperature antiferromagnetic phase in a distorted rocksalt structure with cube axes $a$ $=4.256 \AA$ and $c=4.189 \AA .{ }^{1,3,7}$ The material often contains $\mathrm{N}$ vacancies, and the closely related $\mathrm{Mn}_{3} \mathrm{~N}_{2}$ is again of the
$\mathrm{NaCl}$ structure, but with an ordered array of $\mathrm{N}$ vacancies. ${ }^{1}$ Recent $\mathrm{x}$-ray and neutron diffraction measurements have been performed on $\mathrm{MnN}$ samples prepared as sputtered thin films, in which the $\mathrm{N}$ vacancies common to earlier material appear to be rare. ${ }^{3,4}$ The results confirm the antiferromagnetic phase below a Néel temperature of $660 \mathrm{~K}$, displaying ferromagnetic alignment along the $c$ axis within each $a b \mathrm{Mn}$ plane, and antiferromagnetic order between adjacent planes. The structure reverts to cubic (FCC) above the Néel temperature.

The developing interest in $\mathrm{MnN}$ has encouraged a number of band-structure calculations, ${ }^{8-12}$ which reproduce the observed structures and magnetic phases of $\mathrm{MnN}$. There are, however, no spectroscopic measurements against which the predicted band structures can be tested. Interestingly, to our knowledge there are also no definitive measurements of the dc conductivity of MnN. It is known to be metallic based on 
the value of its ambient-temperature conductivity, in agreement with computed band structures, but no full temperaturedependent conductivity measurements appear to have been reported.

In this paper we describe the preparation of $\mathrm{MnN}$ films by ion-assisted deposition and their characterization by Rutherford backscattering spectroscopy (RBS) supported by nuclear reaction analysis (NRA), and x-ray diffraction (XRD) to establish that they are stoichiometric and of the expected distorted rocksalt structure. Extended X-ray absorption fine structure (EXAFS) measurements will be seen to show no evidence of like-ion nearest neighbors and to demonstrate a high degree of order within the crystallites comprising the films. We then report the first studies of the dc conductivity at temperatures from 4 to $300 \mathrm{~K}$ and of the spectroscopic conductivity from 0.01 to $6 \mathrm{eV}$. These bandstructure probes are complemented with $\mathrm{x}$-ray spectroscopic measurements of partial densities of filled and empty states (PDOS).

Calculations of the interband contribution to the optical conductivity of single-crystalline $\mathrm{MnN}$ are carried out using the linearized muffin-tin orbital (LMTO) method. The predictions of these are appropriately angle-averaged to represent the isotropic spectral conductivity of polycrystalline films, and the results are compared with the measured spectrum. Disentangling the intraband (Drude-type) contribution is not straightforward, but nonetheless we will show that it is possible to associate definite experimental features with corresponding theoretical features. There are discrepancies, in especially the strength of various interband features, that result from the nanocrystalline nature of the films. The imaginary part of the dielectric function is further analyzed in terms of separate band-to-band contributions, and the dominant nature of the main experimentally observed peaks in terms of specific $k$-point regions in the Brillouin zone are determined. Polarization dependences are predicted, although these cannot be verified with the polycrystalline films in this study.

\section{EXPERIMENTAL AND COMPUTATIONAL METHODS}

\section{A. Sample preparation and characterization}

We have grown $\mathrm{MnN}$ films by an ion-assisted deposition process, depositing Mn from a thermally heated source while exposing the substrate to a $500 \mathrm{eV}$ nitrogen ion beam from a Kaufmann-type source. The growth rate was $1 \AA / \mathrm{s}$, and the ionised N:Mn rates were held at 2:1 (ion current density $90 \mu \mathrm{A} \mathrm{cm}^{-2}$ ) to encourage growth of the most N-rich phase, $\mathrm{MnN}$. The films were grown to a thickness of between 150 and $200 \mathrm{~nm}$. To ensure clean growth conditions the vacuum chamber was pumped to $5 \times 10^{-6} \mathrm{~Pa}$ prior to deposition. The substrates were first sputtered with nitrogen to prepare the surface and improve adherence, and thereafter were held at ambient temperature throughout the deposition. Substrates of silicon (100), fused silica, and glassy carbon were used, and we have found no evidence of differences among the films on these various substrates.

The surface of the films were inspected by scanning electron microscopy (SEM) and found to be smooth. Their com-

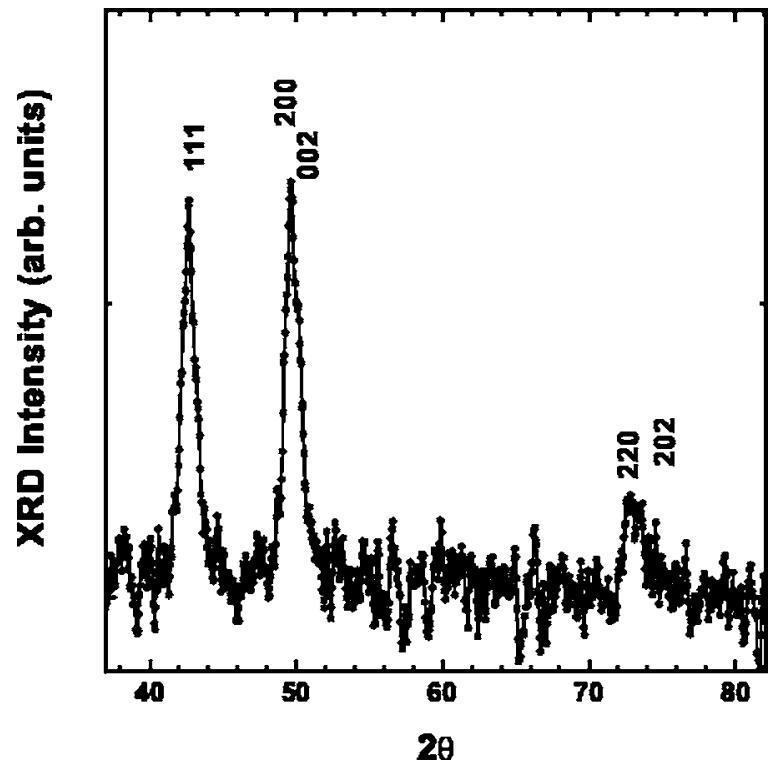

FIG. 1. XRD data for $\mathrm{MnN}$ deposited by ion-assisted deposition, demonstrating the nanocrystalline distorted $\mathrm{NaCl}$ structure of the films.

position was established primarily by RBS, complemented for lighter elements $(\mathrm{N}, \mathrm{O})$ by NRA. All films were found to be stoichiometric $\mathrm{MnN}$ to within the 1 to 2 at. \% accuracy of the measurements. The area density of the atomic species provided by RBS and NRA was also used to confirm the film thicknesses measured by a quartz microbalance during growth.

Structural characterization was accomplished by XRD and EXAFS. Turning first to XRD we show in Fig. 1 the pattern provided by a grazing-incidence geometry on a film grown on $\mathrm{Si}$. All of the features can be assigned to the known distorted $\mathrm{NaCl}$ structure of $\mathrm{MnN}$, and the width of the strongest (111) peak indicates an average crystallite diameter of about $8 \mathrm{~nm}$. At that crystallite size it is not possible to resolve the (200) and (002) peaks fully, but the clear evidence of an asymmetry in that feature confirms the presence of a distortion along the $c$ axis, as expected in the antiferromagnetic phase. ${ }^{3}$ The peak positions are in full agreement with the unit cell dimensions for rocksalt $\mathrm{MnN}$, further confirming their 1:1 Mn:N stoichiometry. In particular we note that there is no evidence at all of the peaks of $\mathrm{Mn}_{3} \mathrm{~N}_{2}$, a common secondary phase in $\mathrm{MnN} .{ }^{1,4}$

These structural parameters are confirmed by EXAFS measurements performed on the spectrometer EXAFS-13, line D42, at LURE, Paris Sud University. Figures 2(a) and 2(b) show the Mn $K$-edge EXAFS pattern and the pseudoradial-distribution function (Fourier transform of the EXAFS) for the first four nearest-neighbor shells extracted from an x-ray absorption spectrum taken at $77 \mathrm{~K}$. The parameters obtained from the fit (solid lines in Fig. 2) are compared in Table I with the crystalline values reported at room temperature. ${ }^{3}$ The EXAFS, which has been simulated using a single peak, is used to fit each shell (i.e., a cubic rocksalt structure is assumed) and the fitted lattice constant is found to be $4.195 \AA$, intermediate between the $a b$ and $c$ axis lattice constants of tetragonal MnN. Thus, the results show only 

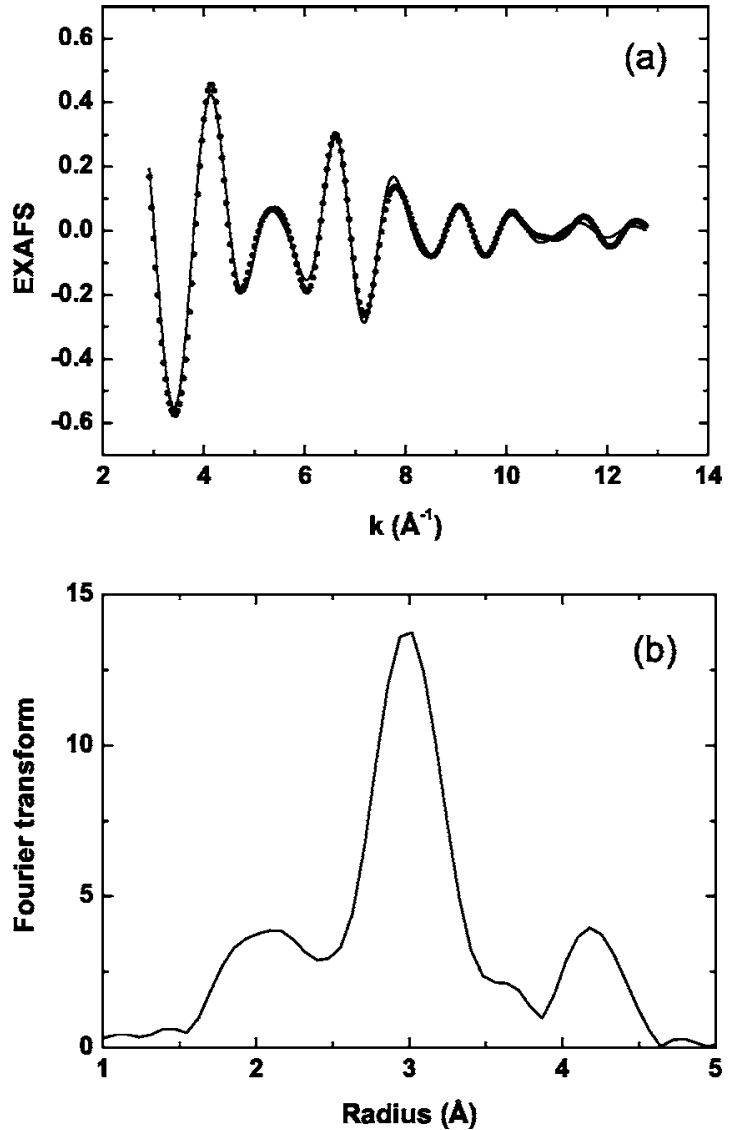

FIG. 2. (a) Extended x-ray absorption fine structure for MnN. (b) Fourier transformed EXAFS showing the first four nearestneighbor positions.

$\mathrm{MnN}$ to be present, with the interatomic distances and the number of ions within the shells consistent with the expected values. Note, however, that the limited resolution of the EXAFS results prevent detection of the noncubic distortion seen in XRD.

\section{B. Electrical and spectroscopic measurements}

The temperature dependent electrical resistivity was measured on a channel scribed on the films to form a conventional four-terminal geometry. The spectral conductivity was determined by ellipsometry (Beaglehole Instruments Picometer) across the visible-UV $(1.5-6 \mathrm{eV})$, and by KramersKronig analysis of reflectance measurements through the mid-IR-visible [Bomem DA8 Fourier-transform infrared

TABLE I. Comparison between lattice parameters extracted from EXAFS measurements and literature values for $\mathrm{MnN}$.

\begin{tabular}{ccc}
\hline \hline & This work & Suzuki et al. (Ref. 3) \\
\hline$a(\AA)$ & 4.195 & 4.256 \\
$c(\AA)$ & 4.195 & 4.189 \\
$c / a$ & 1 & 0.9843 \\
$d_{\mathrm{Mn}-\mathrm{N}}(\AA)$ & 2.098 & $2.128(a) / 2.095(c)$ \\
\hline \hline
\end{tabular}

(FTIR) spectrometer and high-accuracy spectrophotometer]. Extrapolations of the reflectivity for the Kramers-Kronig analysis followed a power-law $\omega^{-0.75}$ for frequencies $40000-150000 \mathrm{~cm}^{-1}$ and $\omega^{-4}$ beyond that, chosen to reproduce the ellipsometry-determined UV conductivity, and the standard Hagen-Rubens form $(1-A \sqrt{\omega})$ appropriate for a metallic response at low frequencies. It should be noted that in the overlap region from 1.5 to $4 \mathrm{eV}$ the measured reflectance was in full agreement with that generated from the ellipsometrically determined optical constants, implying that the films are fully specular up to at least $4 \mathrm{eV}$ (33000 $\left.\mathrm{cm}^{-1}, 300 \mathrm{~nm}\right)$.

X-ray absorption near-edge spectroscopy (XANES) was performed at both the $\mathrm{N} K$-edge and the Mn $L$-edges, providing the partial density of filled states projected onto the $\mathrm{N}(2 p)$ and $\mathrm{Mn}(3 d)$ orbitals. In addition soft x-ray emission spectroscopy (XES) was performed at the N $K$-edge, which then gives the $\mathrm{N}(2 p)$ partial density of filled states. The synchrotron experiments were performed at the soft x-ray undulator beamline 511 at MAXlab (Lund, Sweden), which is equipped with a spherical grating monochromator. ${ }^{13} \mathrm{XES}$ results were recorded using a Nordgen-type grazing-incidence spherical grating spectrometer. ${ }^{14}$ The energy resolution of the $\mathrm{N} K$-edge soft x-ray emission spectra presented here is approximately $0.6 \mathrm{eV}$. XANES measurements were performed with incident resolutions of $0.2 \mathrm{eV}$ and $0.1 \mathrm{eV}$ at the $\mathrm{N}$ $K$-edge and Mn $L$-edges, respectively. XANES spectra were recorded using the sample drain current technique to obtain the total electron yield (TEY) and with incoming radiation incident at $75 \mathrm{deg}$ to the sample surface normal. All synchrotron measurements were performed with the sample in an ultrahigh vacuum environment with base pressure 5 $\times 10^{-8} \mathrm{~Pa}$.

\section{Computational approach}

The calculations of the band structure and optical response functions were carried out using the linearized muffin-tin orbital (LMTO) method in the atomic sphere approximation (ASA). ${ }^{15}$ The potential in this method is constructed self-consistently within the density functional theory in the local spin density approximation ${ }^{16}$ using the Ceperley-Alder ${ }^{17}$ exchange-correlation functional as parameterized by Perdew and Zunger. ${ }^{18}$ The results for the band structure obtained in this manner are in good agreement with the full-potential (FP-LMTO) calculations by Lambrecht et $a l .{ }^{9}$ The imaginary part of the dielectric function is then calculated using the approach described in Ref. 19 within the random phase approximation (RPA) and neglecting local field and excitonic effects:

$$
\begin{gathered}
\varepsilon_{2}^{j}(\omega)=\frac{e^{2}}{m^{2} \omega^{2} \pi} \sum_{v, c} \int_{B Z} d^{3} k\left|\left\langle v \mathbf{k}\left|p^{j}\right| c \mathbf{k}\right\rangle\right|^{2} \\
\delta\left(E_{c \mathbf{k}}-E_{v \mathbf{k}}-\hbar \omega\right),
\end{gathered}
$$

where $p^{j}=-i \hbar \nabla_{j}$ is the $j$ th Cartesian component of the momentum operator, and $E_{v \mathbf{k}}$ and $E_{c \mathbf{k}}$ are the occupied valence and unoccupied conduction band states, respectively. A well 


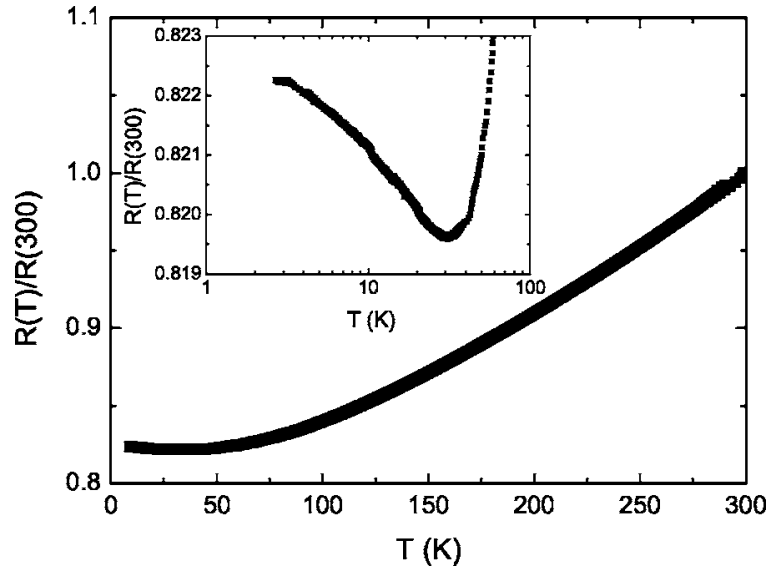

FIG. 3. Normalized temperature dependent resistivity of MnN. The room temperature resistivity is about $135 \mu \Omega \mathrm{cm}$. The inset shows the approximately logarithmic resistance anomaly observed at low temperature.

converged $10 \times 10 \times 7 \mathrm{k}$-point mesh in the reciprocal space unit cell is used in conjunction with the tetrahedron integration method ${ }^{20}$ to evaluate the integral over the Brillouin zone. $\varepsilon_{2}(\omega)$ is calculated up to $20 \mathrm{eV}$ by including all occupied valence bands and up to 40 bands in the conduction band. The calculations of the optical functions are performed for the antiferromagnetic unit cell. From this the real part of the dielectric function is obtained by Kramers-Kronig transformation and any other desired optical function such as the optical conductivity $\operatorname{Re}\{\sigma(\omega)\}=4 \pi \operatorname{Im}[\varepsilon(\omega) / \omega]$ can easily be obtained.

\section{RESULTS}

\section{A. Experiment and comparison to calculations}

Figure 3 displays the temperature dependence of the normalized resistivity. The room temperature resistivity is approximately $135 \mu \Omega \mathrm{cm}$. The films are clearly metallic, though they show a high residual resistivity of more than $100 \mu \Omega \mathrm{cm}$ as expected for the very small crystallites in the films. The intrinsic temperature-dependent resistivity is similarly large, rising to $25 \mu \Omega \mathrm{cm}$ when the temperature reaches $300 \mathrm{~K}$. The inset to Fig. 3 shows a weak approximately logarithmic upturn below $30 \mathrm{~K}$ reminiscent of a Kondo anomaly, which is a feature usually associated with magnetic impurity scattering. ${ }^{21}$ The interpretation of this upturn is as yet unclear.

The measured spectral conductivity (Fig. 4, solid blue line) shows a conductivity that rises to $7500 \mathrm{~S} / \mathrm{cm}$ at the lowest measured frequency, in good agreement with the dc value of $7400 \mathrm{~S} / \mathrm{cm}$. This behavior is characteristic of an intraband metallic contribution, although the data do not fit well to the simple Drude expression

$$
\sigma(\omega)=\frac{\sigma_{D C}}{1+\omega^{2} \tau^{2}} .
$$

As discussed below there are significant low-frequency interband contributions which overlap the intraband conductivity.

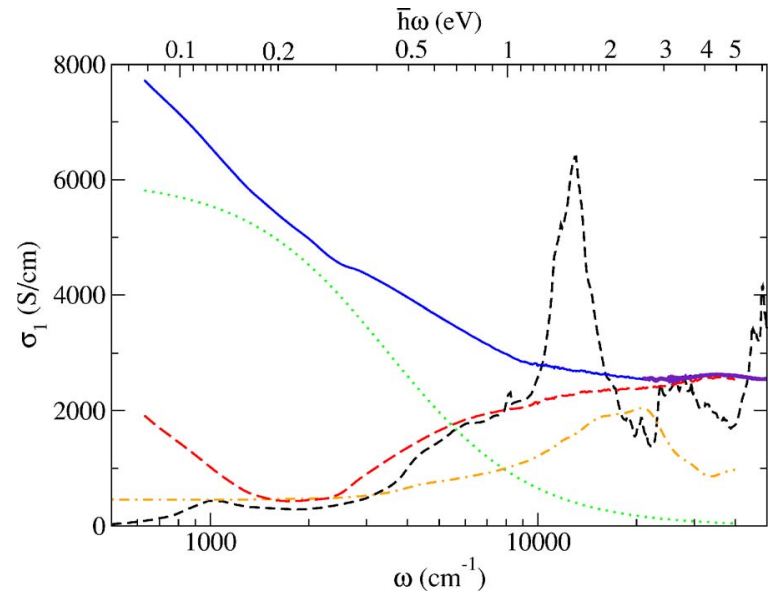

FIG. 4. (Color online) Optical conductivity as the function of a wavenumber and photon energy: Solid blue line experimental data obtained from reflectance and Kramers-Kronig analysis in the infrared, indigo part at higher energy was obtained separately from ellipsometry in the UV, visible and near-infrared; short-dashed black line, calculated interband contribution to the conductivity, dotted green line, Drude-expression with $\sigma(0)=6000 \mathrm{~S} / \mathrm{cm}$ and $1 / \tau$ $=3500 \mathrm{~cm}^{-1}$, long-dashed red line, experiment with Drude interband contribution subtracted, dash-dotted orange line, convolution of empty and filled density of states multiplied by frequency and an arbitrary factor.

In particular, the calculated interband conductivity, (short dashed black line in Fig. 4) shows a feature at $1000 \mathrm{~cm}^{-1}$ or $0.13 \mathrm{eV}$. Therefore, a Drude term, shown as a dotted green line, with $\sigma(0)=6000 \mathrm{~S} / \mathrm{cm}$ and $\tau=9.5 \times 10^{-13} \mathrm{~s}$ was subtracted so as to give approximate agreement between calculated and experimental $\sigma$ in the region $1200-2200 \mathrm{~cm}^{-1}$. The experimental spectrum after the Drude term subtraction, shown as a long dashed red line, then agrees remarkably well in absolute value and shape of the curve all the way up to about $9000 \mathrm{~cm}^{-1}$ or $1.1 \mathrm{eV}$.

At frequencies above $5000 \mathrm{~cm}^{-1}(0.6 \mathrm{eV})$ the conductivity is dominated by interband transitions. In our measured data these are signalled by features with maxima at $14000 \mathrm{~cm}^{-1}(1.7 \mathrm{eV})$ and $32000 \mathrm{~cm}^{-1}(4.0 \mathrm{eV})$. For comparison between the experimental and theoretical data the calculated interband contribution has been averaged over both polarizations, corresponding to the sample's polycrystalline nature, i.e., $\sigma_{a v}=\left(2 \sigma_{a b}+\sigma_{c}\right) / 3$. The strongest peak in the calculation corresponds well in energy to the observed $1.7 \mathrm{eV}$ experimental feature, and a second peak appears in the calculation at $3.4 \mathrm{eV}$, near to the measured feature at $4.0 \mathrm{eV}$. However, both features are much weaker and broader in the experiment than in the theory. Thus there is substantial agreement in regards to the energy at which the structure occurs, but a severe disagreement in the strengths of the features. We now discuss the role that the nanocrystallinity may play in that disagreement.

As will be discussed in the next section, the $1.7 \mathrm{eV}$ peak corresponds to a strong peak in the joint density of states originating from nearly parallel, but not flat, bands across an extended portion of the Brillouin zone (BZ). The calculated conductivity is appropriate for fully crystalline material, and 
thus assumes vertical transitions, or strict momentum conservation. As discussed above, the films used in the experiment comprised crystallites of typically $8 \mathrm{~nm}$ diameter, limiting the mean free path $(\Lambda)$ to about $4 \mathrm{~nm}$. Furthermore it is likely, given the high residual resistivity, that the mean free path is limited further by defects within the crystallites. In the presence of such strong scattering the wavevector of both initial and final states will be broadened to about $\Delta k \sim \Lambda^{-1}$, in turn broadening the interband absorption features by as much as twice the product of the wavevector broadening and the band dispersion. With the band calculated dispersions (see next section) one should expect broadening of at least $0.5 \mathrm{eV}$ from the limited mean free path. The effect on the relatively narrow $1.7 \mathrm{eV}$ feature is especially severe, broadening it by a factor of at least 3 with the accompanying reduction in its strength. It is, however, insufficient to fully explain the reduction to the measured feature.

It is useful further to consider the extreme short mean free path limit, when wavevector conservation is completely violated. In that limit one might expect that the conductivity is proportional to a convolution of the filled and empty parts of the density of states, i.e.,

$$
\sigma_{1}(\omega) \propto \frac{1}{\omega} \int f(E) g(E)[1-f(E-\hbar \omega)] g(E-\hbar \omega) d E,
$$

where $\sigma_{1}$ is the real part of the conductivity, $f(E)$ is the Fermi function, and $g(E)$ the density of states. We can demonstrate the dominant nature of momentum conservation in the $1.7 \mathrm{eV}$ feature by plotting the above convolution multiplied by an arbitrary factor for comparison with the measured spectral function. This curve is shown as the dashdotted orange line in Fig. 4. One may notice that the peak at $1.7 \mathrm{eV}$ is totally absent. In fact, this curve gradually rises and peaks near $2.8 \mathrm{eV}$. If one assumes that the real measured spectrum is some superposition of momentum conserving regions and momentum relaxed regions lying across grain boundaries, one may well imagine that the features get strongly reduced because the peaks in one curve occur near the valleys in the other. The convolution curve on the other hand would strongly underestimate the experimental conductivity in the region below $1 \mathrm{eV}$. As will be shown in the next section, the peak centered at about $0.6 \mathrm{eV}$ arises from smaller regions of the $\mathrm{BZ}$ which are perhaps less affected by the momentum conservation relaxation.

As for the discrepancy in the higher energy peak position, we note that LDA will tend to underestimate conduction band energies and overestimate valence band energies, and thus underestimate optical transition energies. This effect is similar to the well-known underestimate of the band gap in semiconductors. On the other hand, excitonic effects, not included here, would tend to lower optical transitions. Since this is a metallic system, excitonic or electron-hole interaction effects are expected to be strongly screened.

Figure 5 displays both XANES and XES results for N $K$-edge transitions, representing the filled (XES) and empty (XANES) $\mathrm{N}(2 p)$ partial density of states. The XES spectrum was obtained using $419 \mathrm{eV}$ excitation. Note that these techniques do not assign an absolute scale to the PDOS function,

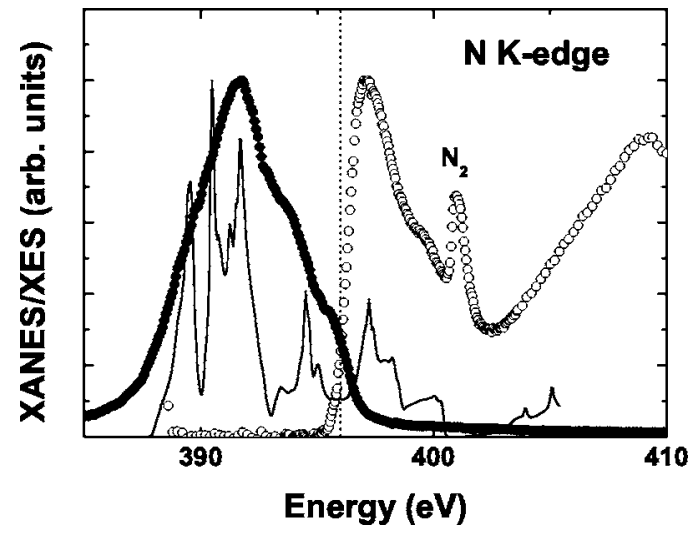

FIG. 5. XANES $(\bigcirc)$ and XES $(\bullet)$ spectra for $\mathrm{N}(1 s)-(2 p)$ transitions representing empty and filled states respectively, compared to calculated N(2p) PDOS (solid line). The narrow peak at $401 \mathrm{eV}$ is due to a small quantity of molecular $\mathrm{N}_{2}$ trapped within the films.

and the two sets of results have simply been normalized to their peak values. On the other hand their energy scales are well-calibrated so that a common Fermi energy for the $\mathrm{MnN}$ is clearly seen at $397 \mathrm{eV}$ (relative to the $1 s$ level), where the XES (filled) DOS falls to zero and the XANES (empty) DOS rises rapidly. The figure also shows the PDOS as predicted by the calculated band structure. The agreement in peak positions is reasonable taking into account the broadening of the experimental results due to both experimental resolution effects and the nanocrystallinity of the sample and the fact that no matrix element energy dependence was taken into account in the calculations. The additional peak at $402 \mathrm{eV}$ in the XANES data is the $\pi^{*}$ resonance of a small density of trapped molecular $\mathrm{N}_{2}$ in the films, as seen also in other IADgrown films. ${ }^{22,23}$ A corresponding $\mathrm{N}_{2} 3 \sigma_{g}$ resonance was also observed in the XES spectrum when the excitation energy was resonant with the $\pi^{*}$ absorption (i.e., $E_{e x}=401 \mathrm{eV}$ ). The presence of these two features with well-known energies ${ }^{24}$ allows an excellent confirmation of the energy calibration. The molecular nitrogen in the films does also add uncertainty to the interpretation of the RBS measurements, but a comparison of the relative intensity of the $\mathrm{N}_{2}$ peak to similar peaks seen in disordered $\mathrm{GaN}$ films ${ }^{22}$ indicates that the fraction of nitrogen held as molecules is within the RBS uncertainty limits.

Figure 6 shows XANES results from the $\operatorname{Mn}(2 p)$ core level. In this case the PDOS is reproduced some $12 \mathrm{eV}$ above the first edge, representing the spin-orbit splitting of the $2 p_{1 / 2}$ and $2 p_{3 / 2}$ states (the $L_{2} / L_{3}$ edges). Again the agreement with the calculated PDOS is reasonable in terms of peak positions. Both experiment and theory show two peaks followed by a shoulder spaced by about $1 \mathrm{eV}$. The intensities are not expected to match because matrix element effects were not included in the theory.

\section{B. Analysis of calculated spectra}

In this section we analyze the calculated optical spectral functions in terms of the band structure. First, we show the calculated optical conductivities again in Fig. 7, this time 


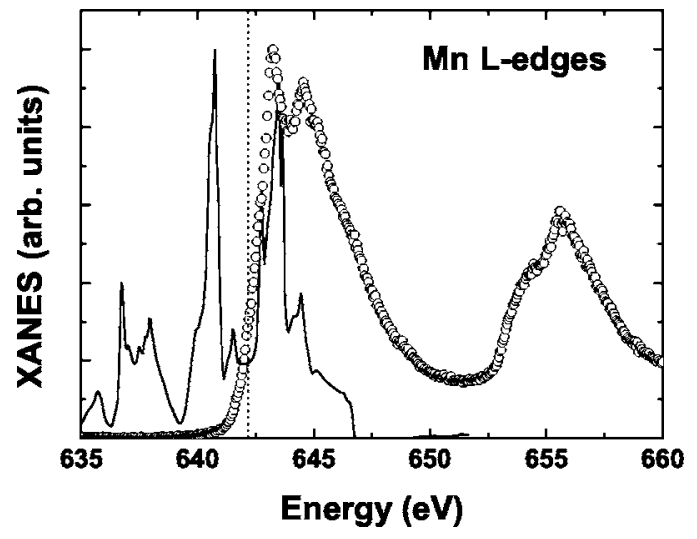

FIG. 6. X-ray absorption near-edge spectrum for the $\operatorname{Mn}\left(2 p_{3 / 2}\right)$ core level $(\bigcirc)$ compared with the calculated $\mathrm{Mn}(3 d)$ PDOS (solid line). The $\mathrm{Mn} L_{2}$-edge $\left(2 p_{1 / 2}\right)$ contributes the features above $652 \mathrm{eV}$.

including the polarization dependence and energies well into the ultraviolet region up to $11 \mathrm{eV}$. Although the polarization dependence could not be determined from the present measurements on polycrystalline films and the present measurements were limited to the IR, visible, and near UV, we hope it will be useful for future reference.

Next, since the primary optical response function calculated is the imaginary part of the dielectric function, we analyze it in partial band to band contributions, beginning in Fig. 8 with the case where the electric field $\mathbf{E}$ is polarized perpendicular to the $c$ axis. For reference, the region of the band structure near the Fermi level is shown in Fig. 9 with some of the bands labeled, counting them upward starting from the $\mathrm{N}(2 s)$-like bands. Bands 13 and 14 are seen to cross the Fermi level.

Returning to Fig. 8 we first note that there is a sharp peak at $0.1 \mathrm{eV}$ which is primarily due to transitions from band 13 to 14 . This arises from the small regions in $k$-space where both bands 13 and 14 cross the Fermi level close to each other, such as a little before halfway along $\Gamma-X$ or along $\Gamma-M$, since of course the transitions only correspond to the region where band 13 is occupied and band 14 empty. Transitions between band 12 and 13 extend from 0 to $2 \mathrm{eV}$ but

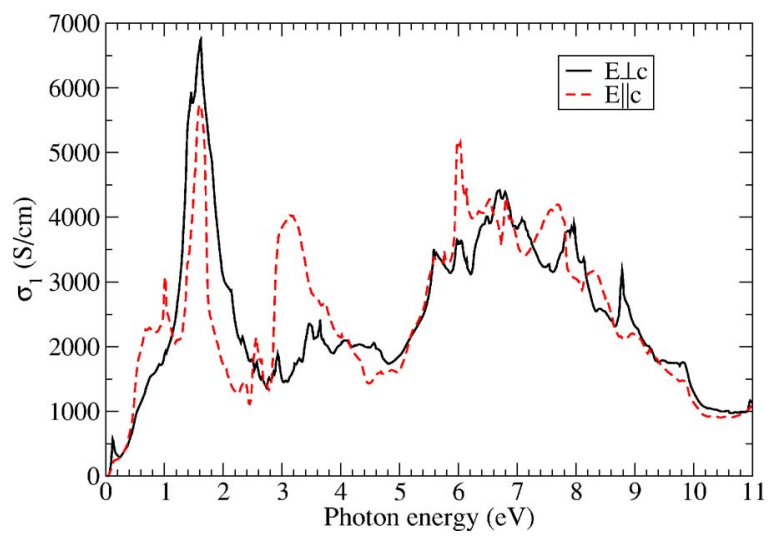

FIG. 7. (Color online) Calculated optical conductivity for incident light polarized along the two inequivalent crystal directions.

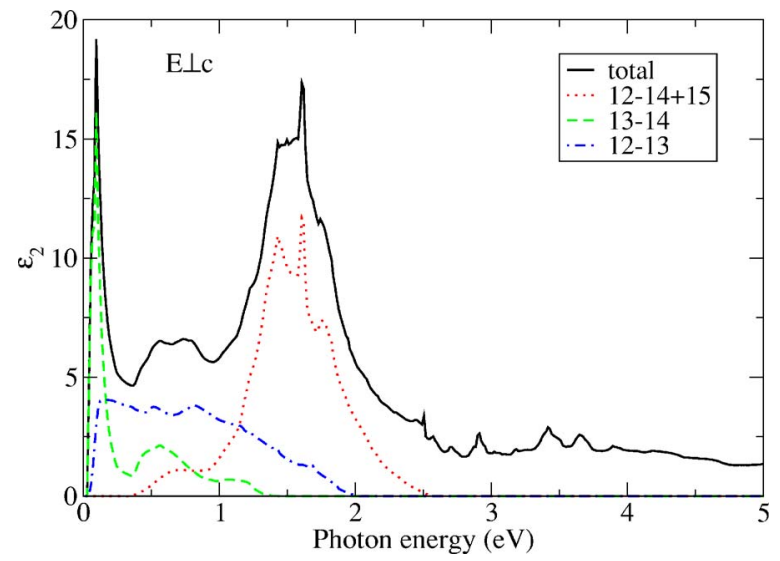

FIG. 8. (Color online) Separate band-to-band contributions to the imaginary part of the dielectric function $\varepsilon_{2}$ of $\mathrm{MnN}$ for $\mathbf{E} \perp \mathbf{c}$.

give a rather broad background-like contribution. The peak standing out at $0.6 \mathrm{eV}$, which corresponds in energy to the onset of strong interband contributions to the measured optical conductivity, is seen to arise from a band 13-14 contribution riding on top of this background. Inspecting the bands, this can be seen to arise from near the $M$ and the $Z$ points, where band 13 is well below the Fermi level and band 14 is above it. Several contributions add together to form the strong peak centered at $1.5 \mathrm{eV}$. These are shown as the dotted red line and correspond to transitions from band 12 to bands 14 and 15 .

Figure 10 shows the energy band differences between bands 14 and 16 from band 12. Whenever bands are parallel, this shows up as flat lines in this plot. This happens for 12-14 along $\Gamma-X$ and mostly for bands $12-15$ over several extended regions in the Brillouin zone all for energies near $1.5 \mathrm{eV}$. This explains why there is a large joint density of states associated with this peak. No other strong features are seen in $\epsilon_{2}(\omega)$ below $5 \mathrm{eV}$.

Next, we turn to the $\mathbf{E} \| \mathbf{c}$ spectrum shown in Fig. 11. First of all, we may notice that the polarization dependence is indeed significant. The main peak (in terms of area under the curve) is now at $0.6 \mathrm{eV}$ with a smaller peak at $1.5 \mathrm{eV}$. The peak above $3 \mathrm{eV}$ which was clearly identified in the experiments is now seen to arise mainly from $\mathbf{E} \| \mathbf{c}$ transitions. The

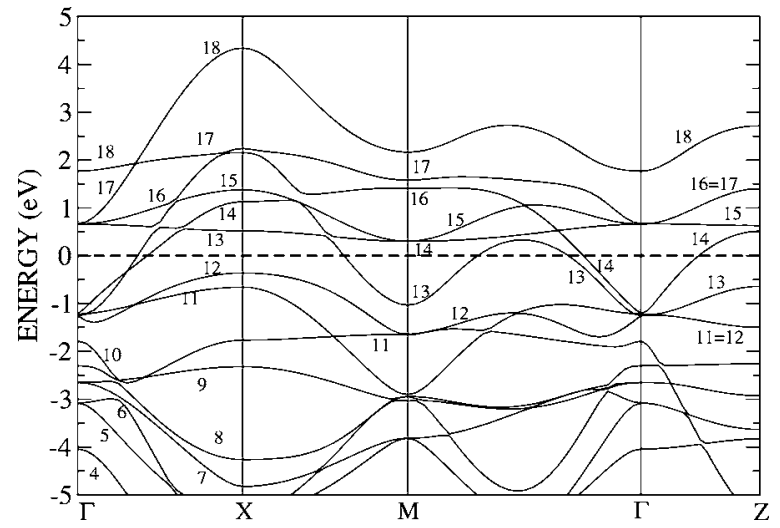

FIG. 9. Calculated band structure of $\mathrm{MnN}$ near the Fermi level. 


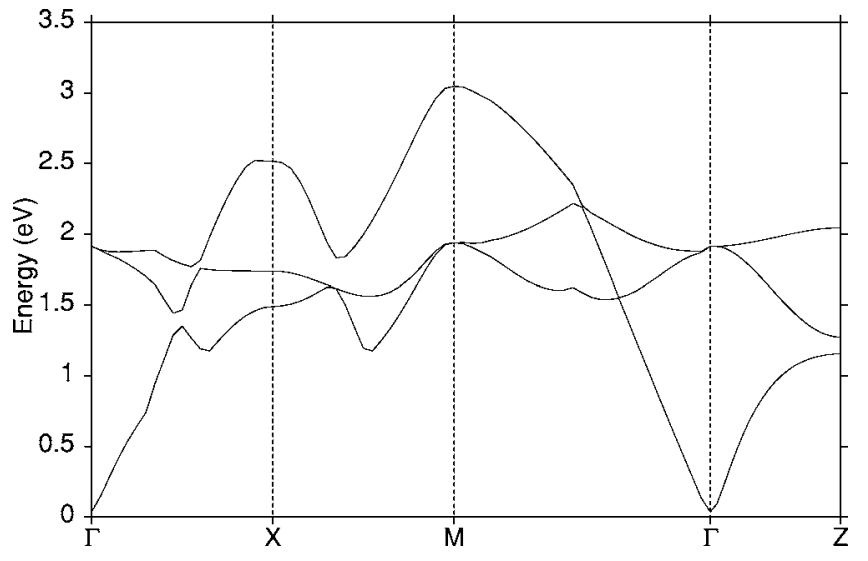

FIG. 10. Calculated energy difference between bands 14 and 16 and band 12 as a function of wave vector.

strong feature at $0.6 \mathrm{eV}$ is seen to arise mainly from transitions between bands 12 and 13 and since there are only a few $\mathbf{k}$-point regions where band 13 is empty, we may associate them with the regions near $X$ and in the middle of the $\Gamma-M$ line. The outstanding contribution to the peak at $1.5 \mathrm{eV}$ is again from transitions between bands 12 and 15 . A small but distinct peak occurring at $2.6 \mathrm{eV}$ can be seen to come mainly from transitions 10 to 15 . The peak starting at $3 \mathrm{eV}$ is seen to arise mainly from 11 to 17 transitions, with additional contributions from 10 to 15 and 10 to 16 . Bands 11 and 17 can be seen to be nearly parallel along large portions of $\Gamma-X$ and $\Gamma-M$ with an energy band difference of about $3 \mathrm{eV}$, explaining the large joint density of states.

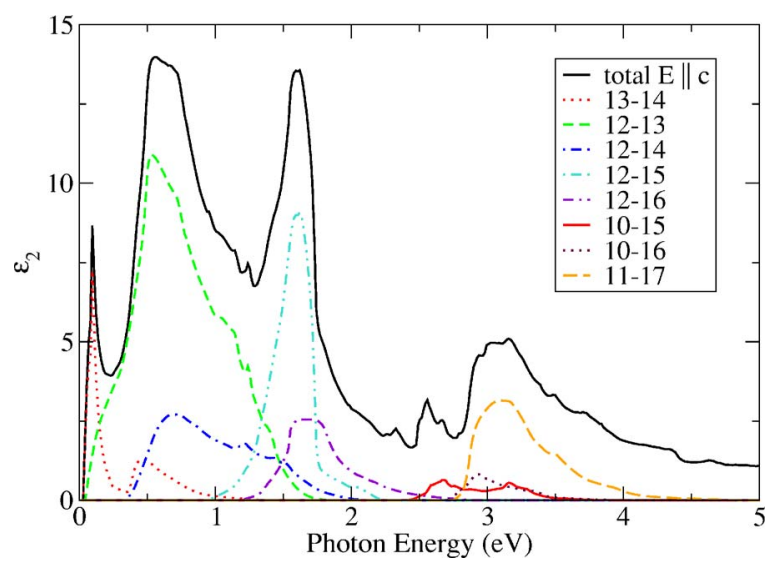

FIG. 11. (Color online) Separate band-to-band contributions to the imaginary part of the calculated dielectric function $\varepsilon_{2}$ of $\mathrm{MnN}$ for $\mathbf{E} \| \mathbf{c}$.

\section{SUMMARY AND CONCLUSIONS}

MnN films were grown by ion assisted deposition. XRD and EXAFS showed them to be nanocrystalline. Temperature dependent dc conductivity measurements were carried out and the optical conductivity in the infrared to visible spectral regions were deduced from reflectivity and ellipsometry measurements. XANES and XES spectra were measured for the N $K$-edge and XANES for the $\mathrm{Mn} L_{1}$ - and $L_{2}$-edges. The XANES and XES spectra were compared to the calculated PDOS of $\mathrm{N}(2 p)$ and $\mathrm{Mn}(3 d)$ character and were shown to be in good agreement when taking into account broadening effects in the measurements. The contribution of interband transitions to the optical response functions were also calculated based on LMTO band structure calculations. Comparison between the calculated and measured optical spectra allowed us to identify certain features in the measured spectra in terms of position of the peaks. Discrepancies in the spectral intensity of the measured and calculated peaks is believed to be due to the nanocrystalline nature of the films which breaks the momentum conservation rule for the optical transitions. As a result features characteristic of large joint density of states due to regions of parallel bands in the calculated spectrum are strongly reduced in the measured conductivity. The calculated and measured conductivity, however, agree nicely in shape and absolute intensity in the spectral region $1000-10000 \mathrm{~cm}^{-1}$ once a Drude like intraband contribution is subtracted from the data. Deviations from Drude like behavior at low energy put in evidence the occurence of interband transitions at low energy, which could furthermore be associated with specific interband transitions between bands 13 and 14 (see Fig. 9) which cross the Fermi level close to each other in several regions of the Brillouin zone. A detailed analysis of the calculated imaginary part of the dielectric function was carried out, thereby assigning the peaks to specific band-to-band transitions and specific regions of the Brillouin zone. This analysis was separately carried out for the two polarization directions and reveals significant polarization dependence.

\section{ACKNOWLEDGMENTS}

The work at V.U.W., I.R.L., and I.G.N.S. was supported by the Tertiary Education Commission through the Centres of Research Excellence Fund. The work at CWRU was supported by the Office of Naval Research through Grant No. N00014-02-1-0880 and the National Science Foundation under Grant No. ECS-0223634. The Boston University program is supported in part by the NSF under Grant Nos. DMR-0311792 and INT-01-04605, by the U.S. ARO under Grant No. 40126-PH, and by the U.S. Air Force Office of Scientific Research.

\footnotetext{
*Present address: Swiss Light Source, Paul Scherrer Institute, CH5232 Villigen PSI, Switzerland.

${ }^{1}$ H. Yang, H. Al-Britten, E. Trifan, D. C. Ingram, and A. R. Smith, J. Appl. Phys. 91, 1053 (2002).

${ }^{2}$ S. Nakagawa and M. Naoe, J. Appl. Phys. 75, 6568 (1994).

${ }^{3}$ K. Suzuki, T. Kaneko, H. Yoshida, Y. Obi, H. Fujimori, and H.
}

Morita, J. Alloys Compd. 306, 66 (2000).

${ }^{4}$ H. Yang, H. Al-Britten, A. R. Smith, J. A. Borchers, R. L. Cappelletti, and M. D. Vaudin, Appl. Phys. Lett. 78, 3860 (2001).

${ }^{5}$ Y. Cui and L. Li, Appl. Phys. Lett. 80, 4139 (2002).

${ }^{6}$ B. K. Rao and P. Jena, Phys. Rev. Lett. 89, 185504 (2002).

${ }^{7}$ A. Leineweber, R. Niewa, H. Jacobs, and W. Kockelmann, J. 
Mater. Chem. 10, 2827 (2000).

${ }^{8}$ M. S. Miao and W. R. L. Lambrecht, Phys. Rev. B 71, 214405 (2005).

${ }^{9}$ W. R. L. Lambrecht, M. Prikhodko, and M. S. Miao, Phys. Rev. B 68, 174411 (2003).

${ }^{10}$ B. R. Sahu and L. Kleinman, Phys. Rev. B 68, 113101 (2003).

${ }^{11}$ A. Janotti, S.-H. Wei, and L. Bellaiche, Appl. Phys. Lett. 82, 766 (2003).

${ }^{12}$ R. de Paiva, J. L. A. Alves, R. A. Nogueira, J. R. Leite, and L. M. R. Scolfaro, J. Magn. Magn. Mater. 288, 384 (2005).

${ }^{13}$ R. Denecke, P. Vaterlein, M. Bassler, N. Wassdahl, S. Butorin, A. Nilsson, J.-E. Rubensson, J. Nordgren, N. Martensson, and R. Nyholm, J. Electron Spectrosc. Relat. Phenom. 101-103, 971 (1999).

${ }^{14}$ J. Nordgren, G. Bray, S. Cramm, R. Nyholm, J.-E. Rubensson, and N. Wassdahl, Rev. Sci. Instrum. 60, 1690 (1989).

${ }^{15}$ O. K. Andersen, O. Jepsen, and M. Šob, in Electronic Band Structure and its Applications, edited by M. Yussouff (Springer, Heidelberg, 1987), p. 1.
${ }^{16}$ P. Hohenberg and W. Kohn, Phys. Rev. 136, B864 (1964); Phys. Rev. 140, A1133 (1965).

${ }^{17}$ D. M. Ceperley and B. J. Alder, Phys. Rev. Lett. 45, 566 (1980).

${ }^{18}$ J. P. Perdew and A. Zunger, Phys. Rev. B 23, 5048 (1981).

${ }^{19}$ W. R. L. Lambrecht, B. Segall, J. Rife, W. R. Hunter, and D. K. Wickenden, Phys. Rev. B 51, 13516 (1995).

${ }^{20}$ P. E. Blöchl, O. Jepsen, and O. K. Andersen, Phys. Rev. B 49, 16223 (1994).

${ }^{21}$ Michael P. Marder, Condensed Matter Physics (Wiley, New York, 2000).

${ }^{22}$ B. J. Ruck, A. Koo, U. D. Lanke, F. Budde, S. Granville, H. J. Trodahl, A. Bittar, J. B. Metson, V. J. Kennedy, and A. Markwitz, Phys. Rev. B 70, 235202 (2004).

${ }^{23}$ B. J. Ruck, A. Koo, U. D. Lanke, F. Budde, H. J. Trodahl, G. V. M. Williams, A. Bittar, J. B. Metson, E. Nodwell, T. Tiedje, A. Zimina, and S. Eisebitt, J. Appl. Phys. 96, 3571 (2004).

${ }^{24}$ P. Glans, P. Skytt, K. Gunnelin, J.-H. Guo, and J. Nordgren, J. Electron Spectrosc. Relat. Phenom. 82, 193 (1996). 